\title{
On the theory and design of a class of PR causal-stable IIR Non-uniform Recombination Cosine Modulated Filter Banks
}

\author{
S. C. Chan and S. S. Yin \\ Department of Electrical and Electronic Engineering \\ The University of Hong Kong, Pokfulam Road, Hong Kong.
}

\begin{abstract}
This paper studies the theory and design of a class of perfect reconstruction (PR) causal-stable nonuniform recombination cosine modulated filter banks (RN CMFBs) with IIR filters. It is based on the RN CMFB previously proposed by one of the author. A PR FIR RN CMFB of similar specification is first designed. The prototype filters of the CMFBs are then model reduced to obtain a nearly PR (NPR) IIR RN CMFB by modifying a model reduction technique proposed by Brandenstein and Unbehauen. With these NPR IIR RN CMFBs as initial guess, PR IIR RN CMFB with very good frequency characteristics can be obtained readily by solving a constrained nonlinear optimisation problem using for example the function fmincom from MATLAB. Design results show that the proposed method is very effective in designing PR RN IIR CMFBs with good frequency characteristics and different system delays.
\end{abstract}

\section{INTRODUCTION}

Perfect reconstruction filter banks (PR FBs) have important applications in speech, audio, image and array processing. The theory and design of uniform PR FBs has been widely studied [1]. In applications such as signal analysis and coding, PR FBs with nonuniform frequency spacing have the potential to offer more flexibility in time-frequency partitioning. This has attracted considerable interests in designing nonuniform FBs [5-7,9]. One useful approach is the indirect method proposed in [9], where certain channels of a uniform FB are merged using the synthesis FB of a recombination FB or TMUX. It was shown recently in [2] that it is possible to achieve PR in recombination FB. Moreover, if the number of channels of the uniform and recombination TMUX are coprime, than the analysis filters of the resulting recombination nonuniform FB (RNFB) admit an equivalent linear time invariant representation (LTI). In other words, the frequency responses of the analysis filters can be optimized directly, which considerably simplifies the design procedure. A class of RNFB based on the cosine modulation filter banks (CMFB) was also proposed. By imposing a simple matching condition on the filter length, RN FIR CMFB with low design and implementation complexities and good frequency characteristics can be obtained. One advantage of the RNFB is that the PR property is structurally imposed as long as the original uniform and recombination filter banks are PR. Furthermore, dynamic recombination of consecutive channels in the original uniform FB by pre-designed TMUXs is possible [9].

In this paper, the theory and design of a class of RN CMFB with IIR filters are studied. Compared to FIR filters, IIR filters have the potential to offer lower system delay, sharper cutoff and high stopband attenuation than their IIR counterparts. However, the design of PR IIR nonuniform CMFBs is complicated by the highly nonlinear objective function and PR constraints. In [3], a method for designing PR uniform IIR CMFB was proposed. The polyphase components of the prototype filter are assumed to have the same denominator so that the PR constraints can be simplified considerably. PR IIR CMFBs with very good frequency characteristics and lower system delay were obtained. The proposed PR IIR RN CMFB is based on the RN CMFB in [2], except that PR IIR CMFBs are now employed for the original uniform FB and the recombination TMUXs. Following [3], we assume that the denominators of the polyphase components of the prototype filter in each CMFB are identical. The main problem lies in the designing such IIR RN CMFBs is that when the number of variables and constraints increases, the optimization procedure is rather sensitive to the initial guess of the prototype filter. To overcome this problem, a PR FIR RN CMFB with similar specification is first designed by the method in [2]. The PR FIR prototype filters are then model reduced to NPR IIR CMFBs by modifying a model reduction technique proposed by Brandenstein and Unbehauen [4]. The resulting NPR IIR RN CMFB has a similar frequency characteristic and reasonably good reconstruction error and it is employed as the initial guess to constrained nonlinear optimisation software such as fmincon from MATLAB for designing the PR IIR RN CMFB. Design results show that both NPR and PR IIR MDFTFBs with good frequency characteristics and different system delays can be obtained readily by the proposed method. It should be note that although the design of PR FIR CMFBs also involves nonlinear constrained optimisation, it is considerably simpler due to the absence of the poles and satisfactory results are usually obtained without much difficulty, unless the filter length is very long. The reason behind modifying the model reduction method in [8] is that the denominator of the polyphase components of the final IIR prototype filter will have identical denominator and it considerably simplifies the PR constraints. Other advantages of the method are that the stability of the model-reduced filter is guaranteed and the IIR filters so obtained closely approximate the properties of the original FIR filter. By using these NPR IIR prototype filters as initial guess to the constrained nonlinear optimiser, significantly better converging speed and reliability over the direct nonlinear optimization is achieved because the pole locations can be approximately located.

The paper is organized as follows: The theory of PR IIR RN CMFBs is introduced in section 2. The design of the PR IIR CMFBs and the modified model reduction technique are given in Sections 3 to 4 . This is followed by a design example and comparison in Section 5 and finally conclusions are drawn in Section 6.

\section{PR IIR RN CMFBs}

Figure 1 shows the recombination nonuniform FB considered in this paper. Consecutive channels of a uniform $M$ channel analysis FB are combined using the synthesis filters of filter bank having smaller number of channels, say $m_{0}$ as shown in the figure. The sampling rate after recombination is reduced by a factor of $\frac{m_{0}}{M}$. For simplicity, only the merging of the first $m_{0}$ channels of an $M$-channel analysis bank are shown. Further merging of consecutive channels can be performed. Let the merged outputs be indexed by an integer $k, k=0, \ldots, K-1$, and $m_{k}$ the number of channels merged at the $k$-th output. For critical sampling, we have $\Sigma_{k=0}^{K-1}\left(m_{k} / M\right)=1$. In the synthesis banks, each merged output will pass through the analysis filters of the recombination FBs and they will be fed to the synthesis bank of the uniform FB for reconstruction. Each synthesis-analysis 
structure, involving $m_{k}$ channel $\mathrm{FB}$, is called a transmultiplexer (TMUX). It was observed in [2] that if the TMUXs are PR, then they only introduce a signal delays of the subband signal of the uniform FB. If these delays are properly compensated in other subbands, then the system is PR if the original uniform FB is PR. Further, if $m_{k}$ and $M$ are coprime to each, then the equivalent analysis filter of the $k$-th output can be written as [2]

$$
\hat{H}_{k}(z)=\sum_{i=0}^{m_{k}-1} H_{l_{k}+i}\left(z^{m_{k}}\right) G_{i}\left(z^{M}\right),
$$

where $l_{k}$ is position of the first sub-channel to be merged for the $k$-th output. If $l_{k}$ is odd, it is necessary to multiply the sequence $(-1)^{n}$ to the merged output to avoid the problem of spectral inversion. It was also shown in [2] that if a matching condition for the PR CMFBs is satisfied, then the problem of spurious response can be suppressed to yield FBs with good frequency characteristics. For FIR CMFBs, this condition can be imposed by choosing the lengths and cutoff-frequencies of the prototype filters and the of the $M$-channel FB and the $m_{k}$-channel TMUX, respectively, as:

$$
\begin{gathered}
\left(L_{m_{k}} / L_{M}\right)=\left(m_{k} / M\right), \\
\left(\omega_{p_{-m_{k}}} / \omega_{p_{-} M}\right)=\left(\omega_{s_{-} m_{k}} / \omega_{s_{-} M}\right)=\left(M / m_{k}\right),
\end{gathered}
$$

where the subscripts $p$ and $s$ stand for passband and stopband cutoff frequencies of the prototype filters. These conditions help to match the magnitude and phase responses in the transition bands of the prototype filters so that the spurious response can be suppressed. By using the model reduction approach, this requirement on the frequency response of the prototype filters is approximately satisfied. When optimising the IIR RN CMFBs, requirement (2) is unnecessary. It should be noted that RN CMFBs without imposing the coprime condition can also be designed $[2,9]$. In this case, the analysis filters will be linearly periodic time varying (LPTV) and it cannot be described as in (1). Fortunately, a matching condition, like (2) and (3), also applies. Due to page limitation, the details are omitted here.

\section{PR IIR Uniform CMFBs}

The analysis and synthesis filters of a type-IV CMFB can be written as:

$$
\begin{aligned}
& h_{k}(k)=2 h(n) \cos \left[\frac{\pi}{M}\left(k+\frac{1}{2}\right)\left(n-\frac{D}{2}\right)+(-1)^{k} \frac{\pi}{4}\right], \\
& f_{k}(k)=2 h(n) \cos \left[\frac{\pi}{M}\left(k+\frac{1}{2}\right)\left(n-\frac{D}{2}\right)-(-1)^{k} \frac{\pi}{4}\right],
\end{aligned}
$$

where $0 \leq k \leq M-1,0 \leq n \leq N-1, h(n), N$, and $D$ are respectively the impulse response, filter length, and delay of the prototype filter.

The analysis and the synthesis filters of an IIR uniform CMFB also can be expressed as (4) except the prototype filter is: $P_{0}(z)=\frac{\sum_{n=0}^{N_{n}-1} a(n) z^{-n}}{\sum_{n=0}^{N_{d}-1} q(n) z^{-n}}$, where $N_{n}$ and $N_{d}$ are respectively the lengths of the numerator and the denominator polynomials of the prototype filter. Let $E_{k}(z)$ be the type-I polyphase component of the prototype filter such that $P_{0}(z)=\sum_{k=0}^{2 M-1} E_{k}\left(z^{2 M}\right) z^{-k}$. Following [3], if $E_{k}(z)$ 's have the same denominator, that is $E_{k}(z)=N_{k}(z) / D(z)$ for $k=0,1, \ldots, 2 M-1$, then the PR condition for the IIR CMFB simplifies to:

$$
\begin{aligned}
& N_{k}(z) N_{2 M-k-1}(z)+N_{M+k}(z) N_{M-k-1}(z)=\beta \cdot z^{-n_{k}} D^{2}(z), \\
& \quad \text { where } k=0,1, \ldots,(M / 2)-1 .
\end{aligned}
$$

In addition, all the roots of $D(z)$ shall remain inside the unit circle to ensure the stability of the analysis filters and the synthesis filters. The objective function is:

$$
\Phi=\int_{\omega_{s}}^{\pi}\left|P_{0}\left(e^{j \omega}\right)\right|^{d} d \omega+\lambda \cdot \int_{0}^{\omega_{1}} \mid\left(H_{0}\left(e^{j \omega}\right) \mid-1\right)^{d} d \omega
$$

where $\omega_{1}$ is the passband cutoff frequency of the first analysis filters and $\lambda$ is a positive weighting factor. $d=2$ corresponds to the least square design criterion. If approximate equip-ripple passband and stopband errors are desired, larger value of $d$, such as 4 , can be selected. The design problem can be formulated as a constrained optimisation problem where (6) is minimized subject to the PR and stability constraints in (5). For FIR CMFBs, $D(z)=1$ and the design problem is considerably simplified.

\section{MODIFIED MODEL REDUCTION}

Since it is much simpler to design a PR FIR CMFB, we propose to obtain an initial guess to the IIR CMFB design problem mentioned above by a model reduction approach. More precisely, the model reduction method proposed in [8] is modified so that the model-reduced prototype filter has identical denominator in its polyphase components. It was found in [4] that the optimal denominator $D(z)$ for approximating a FIR filter $P_{0}(z)$ can be determined using the following iterative procedure:

$$
D^{(k)}(z)=1+\sum_{n=1}^{L_{q}-1} q^{(k)}(n) z^{-n}, D^{(0)}(z)=1 .
$$

Also, defining that:

$$
X^{(k)}(z)=z^{-(L-1)} P_{0}\left(z^{-1}\right) / D^{(k-1)}(z)=\sum_{n=0}^{\infty} x^{(k)}(n) z^{-n}
$$

Then, $q^{(k)}(n)$ can be calculated by minimizing the following objective function:

$$
\begin{aligned}
& F^{(k)}\left(\boldsymbol{q}^{(k)}\right)=\left(\boldsymbol{B}^{(k)} \boldsymbol{q}^{(k)}-\boldsymbol{d}^{(k)}\right)^{T} \cdot\left(\boldsymbol{B}^{(k)} \boldsymbol{q}^{(k)}-\boldsymbol{d}^{(k)}\right), \\
& \boldsymbol{B}^{(k)}=\left[\begin{array}{cccc}
x^{(k)}(0) & 0 & \cdots & 0 \\
x^{(k)}(1) & x^{(k)}(0) & \ddots & \vdots \\
\vdots & \vdots & \ddots & 0 \\
x^{(k)}\left(L_{q}-2\right) & x^{(k)}\left(L_{q}-3\right) & \cdots & x^{(k)}(0) \\
\vdots & \vdots & & \vdots \\
x^{(k)}(L-2) & x^{(k)}(L-3) & \cdots & x^{(k)}\left(L-L_{q}\right)
\end{array}\right], \\
& \boldsymbol{q}^{(k)}=\left[q^{(k)}\left(L_{q}-1\right), \ldots, q^{(k)}(1)\right]^{T} \text { and } \\
& \boldsymbol{d}^{(k)}=-\left[0, \ldots, 0, x^{(k)}(0), \ldots, x^{(k)}\left(L-L_{q}-1\right)\right]^{T} .
\end{aligned}
$$

The $\boldsymbol{q}^{(k)}$ such that $F^{(k)}\left(\boldsymbol{q}^{(k)}\right)$ is the smallest among all the iterations for a sufficiently large value of $k$ is the desired solution. More importantly, the model reduced IIR filter is always stable. The numerator can be determined separately using the least squares criterion. As mentioned earlier, the denominator of the IIR prototype filter should have the form $D\left(z^{2 M}\right)$. If direct model reduction is used, the numerator and denominators have to be multiplied by certain factors and the filter length is thus unnecessarily increased. Another method is to modify the above procedure by assuming the following form for the model-reduced filter:

$$
\hat{P}_{0}(z)=\frac{N(z)}{D\left(z^{2 M}\right)}=\frac{\sum_{n=0}^{L_{p}-1} a(n) z^{-n}}{\sum_{n=0}^{d} q(n) z^{-2 n M}}, q(0)=1,
$$

where $L_{p} \geq 2 d M, d$ corresponds to the non-zero coefficients of $D\left(z^{2 M}\right)$, excluding $q(0)$. The vector $\boldsymbol{q}^{(k)}$ in (9) is then modified as follows:

$$
\boldsymbol{q}^{(k)}=\left[q^{(k)}(2 d M), q^{(k)}(2(d-1) M), \ldots, q^{(k)}(2 M)\right]^{T}
$$

which can be solved by modifying the corresponding rows of $\boldsymbol{B}^{(k)}$ and $\boldsymbol{d}^{(k)}$. According to [4], this modification does not 
violate the stability theorem, which holds for arbitrarily given $X^{(k)}(z)$. Hence, the model-reduced filter in the form of (10) is still stable, provided that $F^{(k)}\left(\boldsymbol{q}^{(k)}\right)$ is the smallest. More detail of this algorithm can be found in [8].

\section{DESIGN PROCEDURE and EXAMPLE}

\section{Design Procedure:}

Given the decimation ratios $\left\{m_{k} / M\right\}, k=0,1, \ldots, K-1$, where $\sum_{k=0}^{K-1}\left(m_{k} / M\right)=1$.

1. Design the $M$-channel and $m_{k}$-channel PR low-delay FIR uniform CMFBs using (5) and (6). The lengths of the prototype filters and the passband and stopband cutoff frequencies should meet the matching conditions (2) and (3) See [2] for more detail.

2. Obtain the corresponding NPR IIR RN CMFB by modelreducing the prototype filters using the modified model reduction method in Section 4.

3. Determine the PR IIR RN CFMB by using the NPR IIR RN CMFB obtained in step 2 as initial guess to the constrained nonlinear optimization problem having (6) as the objective function and the PR and stability constraints in (5) as the constraints. The $M$-channel uniform CMFB is first designed separately. The recombination TMUX is then designed by minimizing the error between $\hat{H}_{k}(z)$ in (1) and the desired response. The objective functions are:

$$
\Phi=\int_{\omega_{s 2}}^{\pi}\left|\hat{H}_{k}\left(e^{j \omega}\right)\right|^{d} d \omega+\lambda \cdot \int_{0}^{\omega_{1}}\left(\left|G_{0}\left(e^{j \omega}\right)\right|-1\right)^{d} d \omega
$$

where $\omega_{1}$ is the passband cutoff frequency of the first synthesis filter of the $m_{k}$-channel IIR uniform CMFB, $\omega_{s 2}$ is the stopband cutoff frequencies of the recombined analysis filter, and $\lambda$ is a positive weighting factor. The second term helps to maintain the flatness of the first analysis filters of the TMUX in its passband, which is necessary for biorthogonal CMFBs.

In this paper, the constrained nonlinear optimisation in steps 1 and 3 are solved using the function Fmincon in MATLAB. Following $[2,9]$, the system delays of the uniform FB and the recombination TMUXs should preferably be $D=2 s M-1$ and $D_{k}=2 s m_{k}-1$, respectively, where the orders of the FIR prototype filters are $2 m M-1$ or $2 m m_{k}-1$ with $s \leq m$ both integers.

\section{Design Example:}

A PR IIR RN CMFB with the following parameters is designed: $M=5, m_{0}=2, m_{1}=3$. The decimation factors are thus $(2 / 5,3 / 5) . m$ and $s$ are chosen as 6 and 5 , respectively. The prototype filters of the 5-, 2-, and 3-channel FIR uniform CMFBs are of length 59,23 , and 35 , respectively and the system delay of the whole system is 78 samples. The passband cutoff frequencies of the prototype filters of the 5-, 2-, and 3-channel uniform CMFBs are $0.02 \pi, 0.05 \pi$ and $0.03333 \pi$, respectively, while the stopband cutoff frequencies are $0.18 \pi, 0.45 \pi$ and $0.3 \pi$. Fig.2 (a) shows the frequency response of PR RN FIR CMFB so obtained. After model reduction, the orders of the prototype filters of the 5-, 2-, and 3-channel IIR uniform CMFBs are 49, 19, and 29, respectively. Note that the orders of the numerator and denominator of the prototype filter after model reduction are the same and it has the form of (10). Fig. 2(b) is the frequency response of the NPR RN IIR CMFB after model reduction. Its reconstruction error is of the order $10^{-3}$. Fig. 2(c) shows the PR RN IIR CMFB obtained after the nonlinear optimisation. To obtain an equi-ripple response, $d$ in (12) is chosen as 4 . As mentioned earlier, the 5-channel PR IIR uniform CMFB is designed separately as mentioned earlier in Section 3 and step 3 of the design procedure. The 2- and 3- channel IIR recombination CMFB TMUX are then designed using (12) as the objective function subject to the stability and PR constraints in (5). Table 1 and Table 2 give out the coefficients of the PR IIR prototypes. It can be seen that from Fig. 2 that the IIR CMFBs has a better stopband attenuation than its FIR counterpart while the system delays are the same. The orders of the IIR filters are also lower than that of the original FIR FB. All the PR FIR and IIR CMFBs have a PR violation of $10^{-15}$. It is also observed that as the system delay and transition bandwidth are reduced, the performance improvement of the IIR CMFB over its FIR counterparts becomes more apparent.

\section{CONCLUSION}

The theory and design of a class of PR causal-stable RN CMFBs with IIR filters are presented. A PR FIR RN CMFB of similar specification is first designed. The prototype filters of the CMFBs are then model reduced to obtain a NPR IIR RN CMFB by modifying a model reduction technique proposed by in [4]. With these NPR IIR RN CMFBs as initial guess, PR IIR RN CMFBs with very good frequency characteristics can be obtained by solving a constrained nonlinear optimisation problem. A design example is given to demonstrate the usefulness of the proposed approach.

\section{REFERENCES}

[1] P. P. Vaidyanathan, Multirate systems and filter banks, Englewood cliffs, NJ: Prentice Hall, 1992.

[2] X. M. Xie, S. C. Chan and T. I. Yuk, "On the theory and design of a class of perfect-reconstruction nonuniform cosine modulation filter-banks," IEEE Int'l Sym. on Circuits and Systems, ISCAS 2002, vol.5, pp. 285-288, May 2002.

[3] J. S. Mao, S. C. Chan and K. L. Ho, "Theory and design of a class of M-channel IIR cosine-modulated filter banks," IEEE $S P L$, vol. 7, no. 2, pp. 38-40, Feb. 2000.

[4] H. Brandenstein and R. Unbehauen, "Least-squares approximation of FIR by IIR filters," IEEE Trans on SP, vol. 46, no. 1, pp. 21-30, Jan. 1998.

[5] F. Argenti and E. Del Re, "Rational sampling filter bansks based on IIR filters," IEEE Trans. on SP., vol. 45, no. 12, pp. 3403-3408, Dec. 1998.

[6] S. J, Akkarakaran and P. P. Vaidyanathan, "New results and open problems on nonuniform filter-banks, Proc. ICASSP'99.

[7] J. Kovacevic and M. Vetterli, "Perfect reconstruction filter banks with rational sampling factors," IEEE Trans. Signal Processing, vol. 41, pp.2047-2066, Jun. 1993.

[8] S. C. Chan, K. M. Tsui and K. W. Tse, "Design of constrained IIR and interpolated IIR filters using a new semidefinite programming based model reduction technique," submitted to EUSIPCO'2004.

[9] R. V. Cox, "The design of uniformly and nonuniformly spaced pseudoquadrature mirror filters," IEEE Trans. on ASSP., vol. ASSP-24, pp. 1090-1096, Oct. 1986.

[10] X. M. Xie, "New design and realization methods for perfect reconstruction nonuniform filter banks," Ph.D. dissertation, The University of Hong Kong, Jan. 2004.

Table 1. Denominators of the polyphase components of the prototype filters of the CMFBs in Fig.2(c)

\begin{tabular}{|lll|}
\hline 1 & $z^{-1}$ & $z^{-2}$ \\
\hline $\begin{array}{l}\text { 5-channel: } \\
1.00000000000000\end{array}$ & 0.12662052000618 & -0.01567588619220 \\
\hline $\begin{array}{l}\text { 2-channel: } \\
\text { 1.0000000000000 }\end{array}$ & -0.00696355012436 & -0.00053421612050 \\
\hline 3-channel: & & \\
1.00000000000000 & 0.00478127887675 & 0.00025244822111 \\
\hline
\end{tabular}


Table 2. Numerators of the polyphase components of the prototype filters of the CMFBs in Fig.2(c).

\begin{tabular}{|c|c|}
\hline 5-channel: (in increasing order of $n$ ) & 2-channel: \\
\hline-0.00009866020574 & 0.00014550134457 \\
\hline 0.00005351533257 & 0.00043134381679 \\
\hline-0.00057454523678 & -0.00115107784206 \\
\hline 0.00013623380622 & 0.00341241045756 \\
\hline-0.00065308946633 & -0.01143242546587 \\
\hline-0.00031065134882 & -0.02916869660806 \\
\hline-0.00081708038261 & -0.01094069851417 \\
\hline 0.00300779073180 & 0.06979928984501 \\
\hline 0.00208003884410 & 0.19263749233792 \\
\hline 0.00205638253153 & 0.28662807992343 \\
\hline-0.00282310409918 & 0.28639451102147 \\
\hline-0.00516053313131 & 0.19166695685494 \\
\hline-0.00882259065851 & 0.06902808201290 \\
\hline-0.01192249181506 & -0.01290596474615 \\
\hline-0.01130923650487 & -0.03229598148102 \\
\hline-0.00982198990611 & -0.01067482173789 \\
\hline-0.00207004849846 & 0.00054120691690 \\
\hline 0.00899307016706 & 0.00038780994871 \\
\hline 0.02381533890376 & -0.00023538940612 \\
\hline 0.04178468649129 & 0.00023460507628 \\
\hline \multicolumn{2}{|l|}{0.06174633006847} \\
\hline \multicolumn{2}{|l|}{0.08123689031043} \\
\hline \multicolumn{2}{|l|}{0.09816444366701} \\
\hline \multicolumn{2}{|l|}{0.11074795397610} \\
\hline \multicolumn{2}{|l|}{0.11777873660448} \\
\hline 0.11764929729081 & 3-Channel: \\
\hline \multicolumn{2}{|l|}{0.11174725195446} \\
\hline 0.10033076598449 & -0.00006301827426 \\
\hline 0.08427582658762 & 0.00057193259283 \\
\hline 0.06757660192270 & -0.00019698674482 \\
\hline 0.05003607194602 & -0.00087369878161 \\
\hline 0.03367868387720 & 0.00102183823842 \\
\hline 0.02219664453481 & 0.00273106620204 \\
\hline 0.01095169889182 & -0.00578739612600 \\
\hline 0.00627017916756 & 0.00057151739853 \\
\hline 0.00368296870705 & -0.01951366628539 \\
\hline 0.00180107728188 & -0.01255351571153 \\
\hline 0.00310207974575 & 0.01442854548079 \\
\hline 0.00810111168415 & 0.05896988993295 \\
\hline 0.00476513134474 & 0.11468521171846 \\
\hline 0.00362937103625 & 0.16693721317074 \\
\hline 0.00292357014054 & 0.19585620587024 \\
\hline 0.00186005310933 & 0.19563189248784 \\
\hline-0.00235430115576 & 0.16527207060715 \\
\hline-0.00292563915567 & 0.11488241013936 \\
\hline-0.00329954426961 & 0.05993980753401 \\
\hline-0.00407340107579 & 0.00121137077298 \\
\hline-0.00528980401050 & -0.01193143245583 \\
\hline-0.00160376525972 & -0.01925791183589 \\
\hline \multirow[t]{8}{*}{-0.00130468664716} & -0.01406364955600 \\
\hline & -0.00249946410723 \\
\hline & 0.00048789575317 \\
\hline & -0.00090109427094 \\
\hline & -0.00013622502292 \\
\hline & -0.00035485827487 \\
\hline & 0.00005959804410 \\
\hline & -0.00009182139847 \\
\hline
\end{tabular}

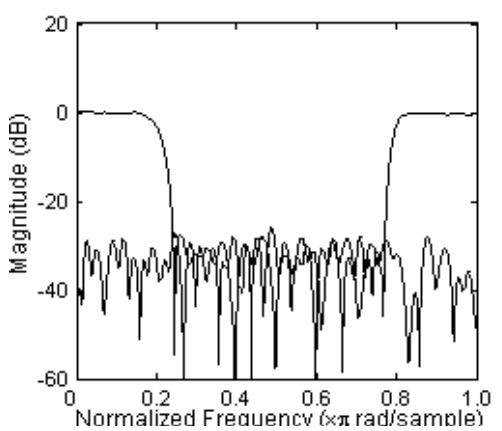

(a)

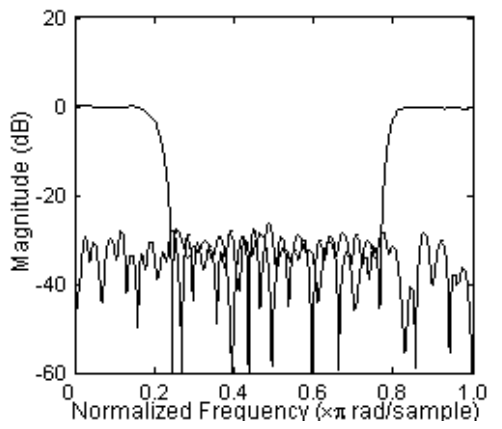

(b)

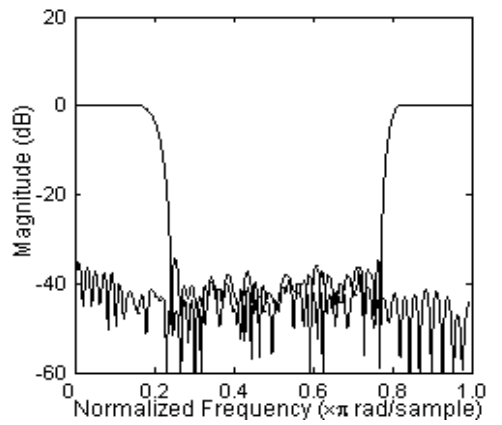

(c)

Fig.2. Frequency responses of the recombination nonuniform CMFBs with decimation ratios $(2 / 5,3 / 5)$.

(a) PR nonuniform PR CMFB with $m=6, s=5$;

(b) NPR IIR nonuniform CMFB after model reduction; (c) PR IIR nonuniform CMFB after nonlinear optimization with (b) as initial guess.

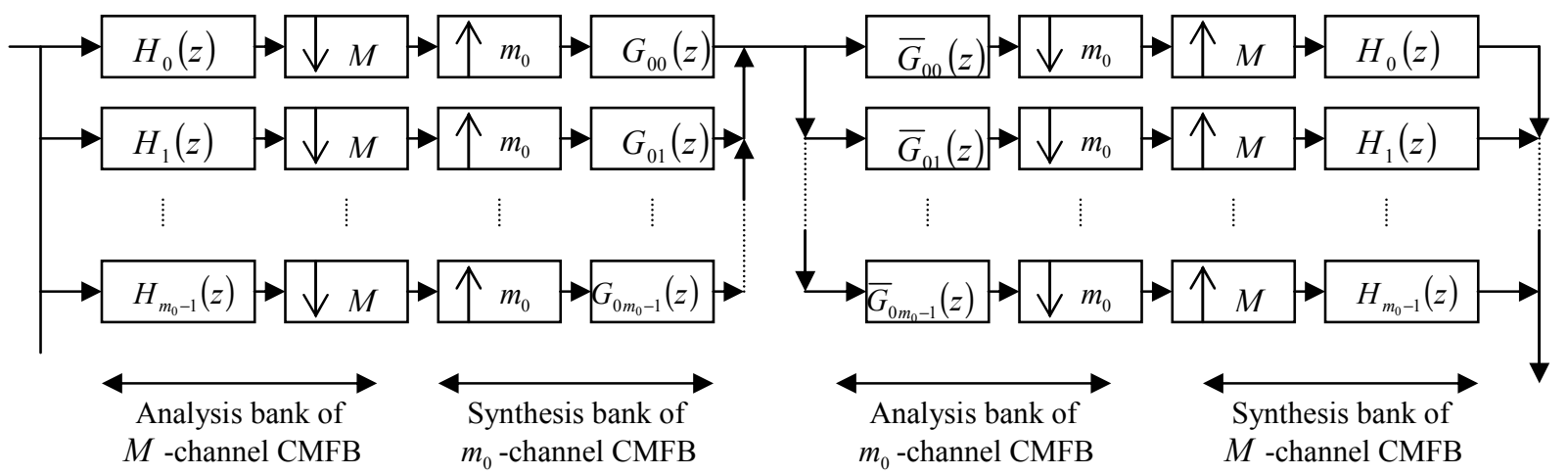

Fig.1. Structure of PR recombination nonuniform CMFB (only the first $m_{0}$ subbands are plotted). 